\title{
Using Transfinite Ordinal Conditional Functions
}

\author{
Sébastien Konieczny \\ CNRS \\ CRIL - Université d'Artois \\ Lens, France \\ konieczny@eril.fr
}

\begin{abstract}
Ordinal Conditional Functions (OCFs) are one of the predominant frameworks to define belief change operators. In his original paper Spohn defines OCFs as functions from the set of worlds to the set of ordinals. But in subsequent paper by Spohn and others, OCFs are just used as functions from the set of worlds to natural numbers (plus eventually $+\infty$ ). The use of transfinite ordinals in this framework has never been studied. This paper opens this way. We study generalisations of transmutations operators to transfinite ordinals. Using transfinite ordinals allows to represent different "levels of beliefs", that naturally appear in real applications. This can be viewed as a generalisation of the usual "two levels of beliefs" framework: knowledge versus beliefs; or rules base versus facts base, issued from expert systems works.
\end{abstract}

\section{Introduction}

Ordinal Conditional Functions (OCFs) [14] are one of the predominant frameworks to represent epistemic state and define belief change operators (see e.g.[14|16,3]1013]). The intuitive appeal of the definition explains its success: an OCF is a function that maps worlds into ordinals. The smaller the ordinal, the more plausible the world for the agent. This representation of epistemic state is more expressive than the one using total pre-orders on worlds, that is one of the canonical ones for classical AGM belief revision [12]3]. The fundamental role of OCF for defining belief revision operators is shown by the fact that Spohn's conditionalization of OCF [14] is often used to illustrate works on iterated belief revision [3[10].

In his original paper Spohn defines OCFs as functions from the set of worlds to the set of ordinals. But in subsequent papers by Spohn [15] and others, OCFs are just functions from the set of worlds to natural numbers (and eventually $+\infty$ ). This restriction is natural, since it is enough to represent usual epistemic states and belief change operators.

But it is strange that in works using OCF it was never studied what the use of transfinite ordinals can bring to the representation of epistemic states, and its consequence on the definition of belief revision operators.

This paper aims at studying transfinite OCFs, i.e. OCFs using transfinite ordinals. Very roughly, transfinite ordinals allow to describe different "infinity levels". From a representational point of view, this allows to encode different "levels of beliefs", i.e. more or less strong beliefs, where the strong ones are considered as integrity constraints by weaker ones.

This allows to define generalisation of usual frameworks. First, when one use OCFs that are defined on the restriction $\langle$ natural numbers $\cup\{+\infty\}\rangle$, the worlds that are

C. Sossai and G. Chemello (Eds.): ECSQARU 2009, LNAI 5590, pp. 396 407, 2009.

(C) Springer-Verlag Berlin Heidelberg 2009 
mapped to $+\infty$ represent unquestionable belief 1 , that are usually called knowledge in this case.

It is a difficult philosophical debate to determine if knowledge exist or not. Which agent can have unquestionable beliefs ? How can an agent be sure that what he "knows" (believes) is absolutely true. It seems to us that no human/artificial agent can be sure of that 2. So speaking of knowledge for a human/artificial agent is just a convenient simplifying convention for designing beliefs much more entrenched than other ones. But, even in these really entrenched beliefs some can be even more entrenched than other ones. So having only one $+\infty$ level to represent deep entrenched beliefs is not enough. One would need to be able to represent differences in the entrenchment of these deep entrenched beliefs.

This distinction between knowledge and belief recalls the traditional view of agent representation in expert systems and in automation, that divides the epistemic state of the agent between a base of rules (that corresponds to knowledge) that is a set of entrenched beliefs (rules) on how the represented system evolves, and a base of facts (that corresponds to beliefs), that is a set of observations made by the agent (through captors for instance).

To illustrate this view let us give an example about a doctor's epistemic state. The doctor has a base of rules, that represents his medical expertise/beliefs, and has a base of facts, that represents the symptoms that he observes on a particular patient (this can be medical analysis, visual observations, etc.).

For most applications this representation is clearly sufficient. And it allows also to illustrate interesting discussions on the status of iterated belief revision.

In most papers iterated belief revision is presented as the process of incorporating successively incoming new evidences. So the main point seems to be that the successive inputs are just more and more recent observations. It is true that an autonomous agent has to be able to do this kind of change, but it is an error to use iterated belief revision operators [3] to do that. Iterated belief revision operators [3] do not allow to incorporate more and more recent observations, but more and more reliable observations. This subject is the starting point of the two interesting papers [9]4, where it is clearly explained that if one wants to incorporate more and more recent information, one has to use prioritised belief merging. Roughly, if the observations incorporation order depends only of recency, and that they can have different reliability, then just store the observations with their degree of reliability, and merge all those observations.

In [4] Dubois identifies three different kinds of revision. We will focus on the two first ones. The first one is the one we just discuss above, that is incorporation of more and more recent observations. This basically corresponds to cases where the base of rule does not change, and where the base of facts increases. So suppose that the doctor receives successively several different medical analysis (that have different reliability). The incorporation of these facts will change the beliefs of the doctor on the disease of the patient, but will not change his medical expertise. This case can be basically handled by classical AGM belief revision [1/7|12] if all the observations are jointly consistent

\footnotetext{
${ }^{1}$ Note that in this case OCFs can be viewed as a semantics for possibilistic logic [5].

${ }^{2}$ Under the hypothesis of his existence, the only agent that could hold real knowledge is a God agent.
} 
(that is the case if the world does not evolve (usual hypothesis in belief revision - otherwise one has to use update operators [118]), and when the captors are reliable (for instance with direct visual observations)). If the observations are not jointly consistent (for instance if some captors/sources are not reliable), then one has to use the prioritised belief merging framework proposed in [9] to this aim.

The second kind of revision identified by Dubois is when the set of rules of the agent has to be changed. Suppose that our doctor goes to a scientific congress and learn new protocols about a specific disease, then he has to change his base of rules. This is the typical use of iterated belief revision à la Darwiche and Pearl [3]: a more reliable piece of information has to be incorporated in our current theory. So typical examples of DP iterated revision should be scientific theory change rather than every day life observations examples with birds.

Some years ago a very interesting paper from Friedman and Halpern already discuss the problems and dangers of developing new technical change operators without specifying their exact application cases (i.e. without giving them an "ontology") [6]. We think that the papers of Dubois [4] and Delgrande-Dubois-Lang [9] is an interesting reminder of this discussion for iterated belief change.

So to sum up Dubois' view in [4], consider that the agent epistemic state is represented by two bases: a base of rules and a base of facts, the base of rules being more important/reliable/entrenched than the base of facts. Then the two kinds of revision are defined by the base that has to be revised. The first one revise the base of facts, the second one the base of rules.

We think that one can go further than that. There is no objective reason to restrict this process to only two bases, one can need to use more levels of beliefs. So we want to define as many bases as needed, and each of this base can be revised differently.

Let us come back to our doctor example. We can not seriously restrict the beliefs of this agent to a base of medical expertise, and a base of facts on the patient. This agent can have other beliefs much more entrenched that his medical expertise, such as basic arithmetics for instance. So we have at least three "level of beliefs": basic arithmetics that is much more entrenched than medical expertise, that is much more entrenched than facts on the patient.

This is the kind of situation that Transfinite Ordinal Conditional Functions allow to represent and handle.

In next section we we give a short refresher on ordinals, and in Section 3 recall the basic definitions of OCF theory change. Then in Section 4 we define Transfinite Ordinal Conditional Functions, that allow to encode different levels of beliefs in an OCF. In Section 5 we show how to define a Transfinite OCF from a set of classical OCFs that represent the different levels of beliefs. In Section 6 we discuss the revision of Transfinite OCFs, and define relative transmutations, that allow to localize the change to the concerned level of beliefs. Finally we conclude in Section 7 .

\section{Naive Ordinal Arithmetics}

In set theory, the natural numbers can be build from sets:

$\mathbf{0}=\{\}$ (the empty set)

$\mathbf{1}=\{\{\}\}=\{0\}$ 
$\mathbf{2}=\{\{\},\{\{\}\}\}=\{0,1\}$

$\mathbf{3}=\{\{\},\{\{\}\},\{\{\},\{\{\}\}\}\}=\{0,1,2\}$

$\mathbf{4}=\{\{\},\{\{\}\},\{\{\},\{\{\}\}\},\{\{\},\{\{\}\},\{\{\},\{\{\}\}\}\}\}=\{0,1,2,3\}$

etc.

So every natural number can be seen as a well ordered set, and the natural order on natural number is given by inclusion of the corresponding sets $(\alpha<\beta$ iff $\alpha \in \beta$ ).

A possible definition of ordinals is that a set $S$ is an ordinal if and only if $S$ is strictly well-ordered with respect to set membership and every element of $\mathrm{S}$ is also a subset of S.

So, starting from $0(\{\})$, and using a successor operation, noted $\alpha+1=\alpha \cup\{\alpha\}$, allows to build the ordinals.

The ordinals that correspond to natural numbers are finite ordinals. The existence of transfinite ordinals is ensured by the axiom of infinity. The first transfinite ordinal is denoted $\omega$. It corresponds to the set of natural numbers $\{0,1,2, \ldots\}$. But we can define a successor to this ordinal $\omega$. So we can define $\omega+1, \omega+2$, etc. until $\omega+\omega=\omega \cdot 2$.

If we describe $\omega$ as the set $\left\{a_{0}, a_{1}, a_{2} \ldots\right\}$, where $a_{0}<a_{1}<a_{2}<\ldots$, then $\omega+1$ can be seen as the set $\left\{a_{0}, a_{1}, a_{2}, \ldots, b_{0}\right\}$, where $a_{0}<a_{1}<a_{2}<\ldots<b_{0}$. See figure 1 for a graphical representation of $\omega^{2}$.

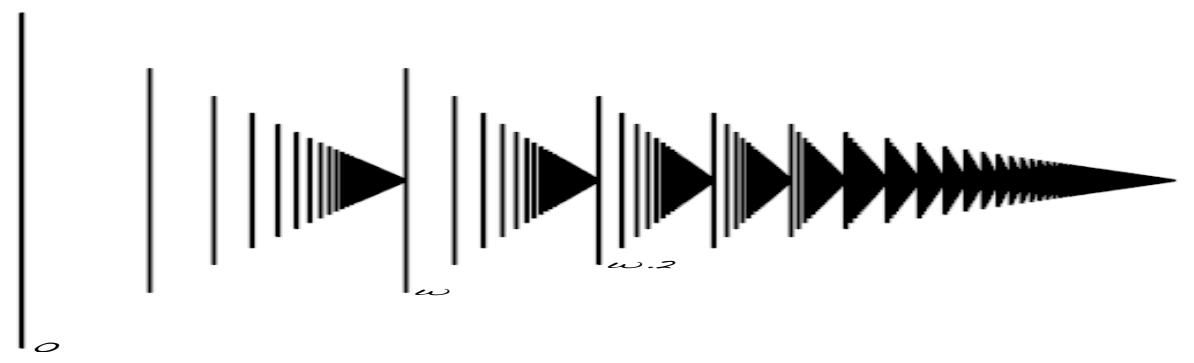

Fig. 1. A graphical "matchstick" representation of the ordinal $\omega^{2}$. Each stick corresponds to an ordinal of the form $\omega \cdot m+n$ where $m$ and $n$ are natural numbers. (Figure from Wikipedia).

Then one can similarly define $\omega .3, \omega .4$, etc. And the ordinal that is the set of all these ordinals is denoted $\omega^{2}$, etc. We will not use ordinals greater than $\omega^{2}$ in this work.

The ordinals $\omega, \omega .2, \omega .3, \ldots, \omega^{2}, \ldots$, that have no predecessor, are called limit ordinals. $\beta$ is a limit ordinal if there is no ordinal $\alpha$ such that $\alpha+1=\beta$.

Let us now define addition on ordinals.

Definition 1. The addition on ordinals $\alpha+\beta$ is defined inductively by:

$-\alpha+0=\alpha$,

$-\alpha+(\beta+1)=(\alpha+\beta)+13$

- if $\beta$ is limit then $\alpha+\beta$ is the limit of the $\alpha+\gamma$ for all $\gamma<\beta$.

\footnotetext{
${ }^{3}$ Recall that " $+1 "$ denotes the successor of an ordinal.
} 
This definition coincides with natural addition when working with finite ordinals. But with transfinite ordinals the addition is not any more commutative. It is for instance easy to see that $3+\omega=\omega$ and is different from $\omega+3$ that is the successor of the successor of the successor of $\omega$.

\section{Classical OCF Theory}

We consider a propositional language $\mathcal{L}$ defined from a finite set of propositional variables $\mathcal{P}$ and the standard connectives.

A world (interpretation) $I$ is a total function from $\mathcal{P}$ to $\{0,1\}$. The set of all worlds is noted $\mathcal{W}$. An interpretation $I$ is a model of a formula $\varphi \in \mathcal{L}$ if and only if it makes it true in the usual truth functional way. $\bmod (\varphi)$ denotes the set of models of the formula $\varphi$, i.e., $\bmod (\varphi)=\{I \in \mathcal{W} \mid I=\phi\}$. Let us denote $\mathcal{O}$ the class of ordinals.

Definition 2. An Ordinal Conditional Function $(O C F) \kappa$ is a function from the set of worlds $\mathcal{W}$ to the set of ordinals such that at least one world is assigned 0.

The meaning of an OCF is simple. The ordinal associated to a world denotes the world plausibility. The higher the ordinal, the less plausible the world. So let us call this the degree of disbelief of the world. In particular world that are assigned 0 are the most plausible worlds, i.e. the currently believed worlds. This means that if one use OCFs as representation of epistemic states for iterated belief revision, the belief base $\varphi$ associated to this epistemic state $\varphi=\operatorname{Bel}(\kappa)$ is defined by those models: $\bmod (\varphi)=\{I \mid \kappa(I)=0\}$. The set of OCFs will be denoted $\mathcal{K}$.

The degree of disbelief can be straightforwardly extended to formulae (set of worlds).

Definition 3. The degree of disbelief of a formula $\varphi$ is the minimum of the degree of disbelief of its models: $\kappa(\varphi)=\min _{I=\varphi} \kappa(I)$.

And one can also define the degree of acceptance of a formula.

Definition 4. A formula $\varphi$ is accepted (for an $O C F \kappa$ ) if $\kappa(\varphi)=0$.

The degree of acceptance of an accepted formula $\varphi$ is $d_{\kappa}(\varphi)=\kappa(\neg \varphi)$.

Now we can define change operators in this setting as functions that change the degree of acceptance of a formula:

Definition 5. A Transmutation [16] is a function that, given an OCF $\kappa$, a formula $\varphi$ and a degree of acceptance $\alpha$, produces a new $O C F \kappa *(\varphi, \alpha)$ such that $\varphi$ is accepted with degree $d_{\kappa *(\varphi, \alpha)}(\varphi)=\alpha$.

Several different transmutation operators can be defined. The problem is to meet the condition of transmutation operators while keeping as much information as possible from the old OCF. As for works on AGM belief revision there are several ways of considering this minimality. The two most usual ones are conditionalization [14] and adjustment [16]. 
Definition 6. The $(\varphi, \alpha)$-conditionalization of $\kappa$ is a new $O C F \kappa *_{C}(\varphi, \alpha)$ defined as

$$
\left(\kappa *_{C}(\varphi, \alpha)\right)(I)=\left\{\begin{array}{l}
-\kappa(\varphi)+\kappa(I) \quad \text { if } I \models \varphi \\
(-\kappa(\neg \varphi)+\kappa(I))+\alpha \text { if } I \models \neg \varphi
\end{array}\right.
$$

where $-\beta+\alpha$ represents the ordinal $\gamma$ such that $\beta+\gamma=\alpha$.

Conditionalization moves all the models of $\varphi$. Adjustment moves only the most plausible models of $\varphi$ (some models of $\varphi$ are moved if necessary).

Definition 7. The $(\varphi, \alpha)$-adjustment of $\kappa$ is a new $O C F \kappa *_{A}(\varphi, \alpha)$ defined as

$$
\left(\kappa *_{A}(\varphi, \alpha)\right)(I)= \begin{cases}0 & \text { if } \kappa(I)=\kappa(\varphi) \\ \alpha & \text { if } I \models \neg \varphi \text { and } \kappa(I)<\alpha \\ \kappa(I) & \text { otherwise }\end{cases}
$$

Adjustment can be seen as the counterpart of Boutilier's natural revision [2] for OCFs.

\section{Transfinite OCF}

So the aim of this work is to encode different "levels of beliefs" in a same OCF. These levels of beliefs have to be strictly hierarchized, in order to ensure that a belief in a higher level is considered as an integrity constraint by the beliefs in lower levels. Let us illustrate this need on a car-driving example.

Example 1. The most important beliefs of the agents are physical beliefs, that compose the highest level of beliefs:

- The road is slippery if and only if it is snowed or frozen $(s l \leftrightarrow s n \vee f)$.

Then the driving behaviour rules form the second level of beliefs:

- If the road is slippery, then adopt a moderate speed $(s l \rightarrow m)$.

- If there are roadworks, then adopt a moderate speed $(w \rightarrow m)$.

The first rule being more important/entrenched/believed than the second one (let us assign a weight of 2 to the first rule, and a weight of 1 to the second one).

Finally the lowest level of beliefs is the one of facts that describe the agent's beliefs about the current situation:

- The road is snowed (sn).

- There are no roadworks $(\neg w)$. The road is not frozen $(\neg f)$.

The belief that the road is snowed is more important/entrenched/believed than the fact that the road is not frozen. Let us assign a weight of 5 to the first fact (the road is snowed), and a weight of 2 to the other ones. The numbers reflect in a sense the intensity of belief of these facts for the agent.

So we will use transfinite ordinals in order to encode the different levels of beliefs. The idea is to use a limit ordinal as boundary between two levels of beliefs. 
Definition 8. A Transfinite $O C F$ is an $O C F \kappa$ such that for any world I either $\kappa(I)=0$ or $\omega .(m-1)<\kappa(I)<\omega . m$, and for at least one world $I^{\prime} \kappa\left(I^{\prime}\right)>\omega$.

When the last inequality hold $m$ is called the level of belief of $I$, and is denoted $\lambda^{\kappa}(I)$. And if $\kappa(I)=0$, then $\lambda^{\kappa}(I)=1$.

The level of belief of a formula, noted $\lambda^{\kappa}(\varphi)$, is the minimum level of beliefs of its models: $\lambda^{\kappa}(\varphi)=\min _{I=\varphi} \lambda^{\kappa}(I)$.

So a Transfinite OCF that corresponds to the car-driving example of example 1 is for instance:

Example 2. Let us introduce our representation of OCF. The ordinal at the left of the line is the one associated to the worlds at the right. The propositional symbols are considered in the order $(f$ sn sl $w m)$ for the interpretations. The notation $*$ represents all the worlds where $*$ can be replaced by 0 or 1 , for instance $1 * 1$ is a shortcut for $\{101,111\}$.

$\kappa$ :

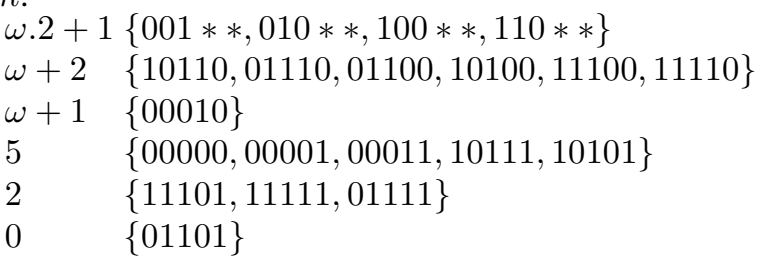

This OCF represents the information given by the rules of Example1 It is obtained in the usual way [5]. So in this example the worlds that are associated to the ordinal 0 are the worlds that satisfy all the formulas of all the levels of beliefs. The worlds that are associated to 2 or 5 are the worlds that satisfy the two most important levels of beliefs, the worlds that are associated to 2 being more plausible than the ones that are associated to 5 . The worlds that are associated to $\omega+1$ and $\omega+2$ satisfy the most important level of beliefs. The worlds that are associated to $\omega .2+1$ do not satisfy the most important level of beliefs.

\section{Building a Transfinite OCF from a Set of Classical OCFs}

It is possible to build a Transfinite OCF from a set of classical OCFs 4 , each classical OCF representing one level of beliefs.

Definition 9. Let $\kappa_{1}, \ldots, \kappa_{n}$ being the classical OCFs that represent respectively the first (least important), ..., last (most important) level of beliefs. Then $\kappa$ is the Transfinite OCF defined inductively as $\kappa(I)=\kappa_{\kappa_{1}, \ldots, \kappa_{n}}(I)$ :

- $\kappa_{\emptyset}(I)=0$

$-\kappa_{\kappa_{1}, \ldots, \kappa_{n}}(I)= \begin{cases}\omega \cdot(n-1)+\kappa_{n}(I) & \text { if } \kappa_{n}(I)>0 \\ \kappa_{\kappa_{1}, \ldots, \kappa_{n-1}}(I) & \text { otherwise }\end{cases}$

\footnotetext{
${ }^{4}$ Let us call Classical OCFs OCFs where all the ordinals associated to worlds are strictly smaller than $\omega$. And let us call Constrained OCFs OCFs where all the ordinals associated to worlds are smaller or equal to $\omega$.
} 
Coming back to the car-driving example, this amounts to consider the three following classical OCFs representing the three levels of beliefs:

Example 3. The first level of belief, containing the facts, is encoded by $\kappa_{1}$. The second one, containing the driving behaviour rules, is encoded by $\kappa_{2}$. The last one, containing physical beliefs, is encoded by $\kappa_{3}$.

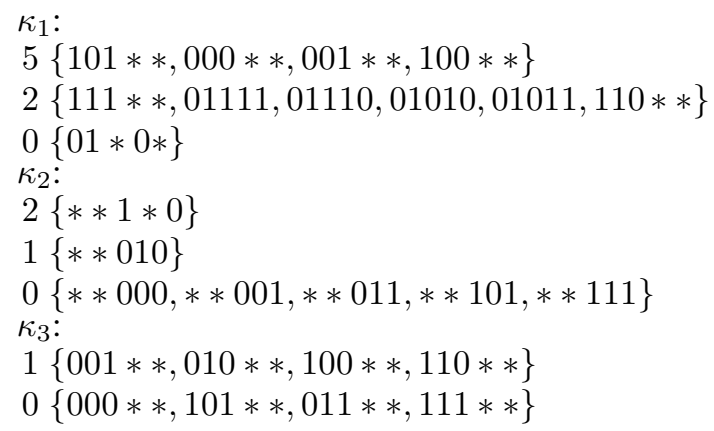

It is easy to check that starting from $\kappa_{1}, \kappa_{2}, \kappa_{3}$ and using the construction of definition 9 , we obtain $\kappa$ of example 2.

\section{Revising Transfinite OCFs}

Of course, since Transfinite OCFs are a subclass of OCFs, then one can use usual conditionalization (or adjustment, or any other transmutation) on Transfinite OCFs.

But this may cause some problems since conditionalization allows to change the degree of acceptance of an interpretation (so of any formula), to any new degree.

This freedom may cause problems for Transfinite OCFs, since this means that this allows to "merge" different levels of beliefs together, just as if there was only one such level. So in this case this means that the representation using levels of beliefs is useless. Since it would be possible for instance to define a classical OCF (using a mapping from the Transfinite OCF) with exactly the same behaviour for transmutations/conditionalization (up to the mapping).

So we would rather need a conditionalization (or more generally a transmutation) that allows only change inside each level of beliefs.

Let us see how to define this operation below. Let us call usual conditionalization (resp. transmutation) absolute conditionalization (resp. absolute transmutation). We will define now relative conditionalization (and relative transmutations).

First, let us illustrate what the Transfinite OCF means from each level of beliefs. This is done using projections.

Definition 10. Let $\kappa$ be a Transfinite OCF with $n$ different levels of beliefs. The $i$ projection of $\kappa$ (projection of $\kappa$ on the $i$-th level of belief), denoted $\kappa_{\downarrow}$ is defined as:

$$
\kappa_{\downarrow i}(I)=\left\{\begin{array}{llr}
\omega & \text { if } \quad \omega . i<\kappa(I) \\
\kappa(I) & \text { if } \omega .(i-1)<\kappa(I)<\omega . i \\
0 & \text { if } \quad \kappa(I)<\omega .(i-1)
\end{array}\right.
$$

So let us see a projection of the Transfinite OCF of the example: 
Example 4. From the most important level of beliefs point of view, the projection is just:

$$
\begin{aligned}
& \kappa_{\downarrow 3}: \\
& 1\{001 * *, 010 * *, 100 * *, 110 * *\} \\
& 0\{000 * *, 101 * *, 011 * *, 111 * *\}
\end{aligned}
$$

The worlds that are associated to 0 in the third level of beliefs, will be eventually discriminated by lower levels.

From the second level of beliefs point of view, the third level of beliefs appear as integrity constraints that can not be questioned, so all the worlds that are not associated to 0 in the (projection of the) third level of beliefs are just impossible worlds. So the projection is:

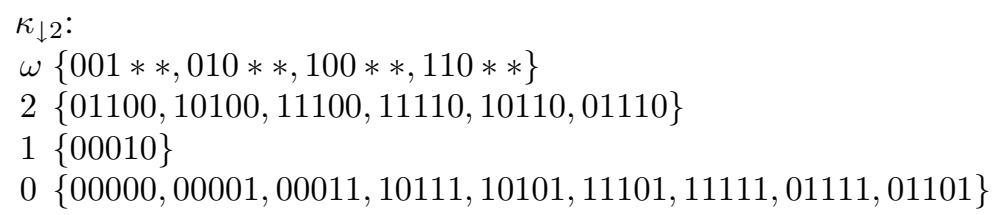

Now from the first level of beliefs, all the highest levels of beliefs appear as integrity constraints. So the projection is:

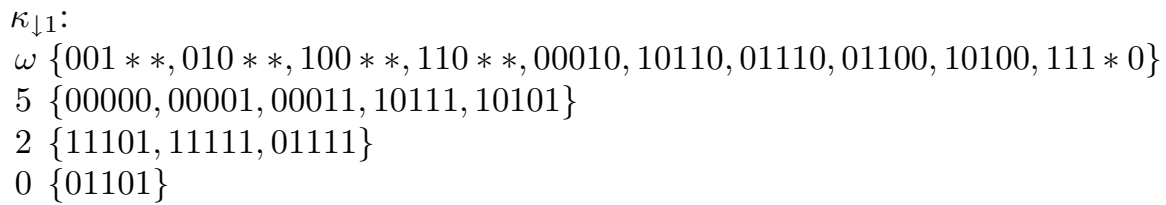

These projection give an idea of how to revise Transfinite OCFs. A relative transmutation will only change the corresponding projection (i.e. level of beliefs). Since if the level of a formula is $i$, this means that a change of its degree of disbelief will change the information of the $i$-th level of beliefs.

Let us define relative transmutation formally.

Definition 11. Let $\kappa$ be a Transfinite OCF with $n$ levels of beliefs. Let $\alpha$ be an ordinal $\alpha<\omega$. Given a (absolute) transmutation $*$. Then the corresponding relative transmutation 娄 is defined as:

$$
(\kappa \circledast(\varphi, \alpha))(I)= \begin{cases}\kappa(I) & \text { if } \kappa^{o}(I)=\omega \\ \kappa(I) & \text { if } \kappa^{o}(I)=0 \text { and } \lambda^{\kappa}(I)<\lambda^{\kappa}(\varphi) \\ \mathbf{B}\left(\kappa, \lambda^{\kappa}(\varphi)-1\right)+1 & \text { if } \kappa^{o}(I)=0 \text { and } \lambda^{\kappa}(I)=\lambda^{\kappa}(\varphi) \\ \omega \cdot \lambda^{\kappa}(\varphi)+\kappa^{o}(I) & \text { otherwise }\end{cases}
$$

where

- $\kappa^{o}=\kappa_{\downarrow \lambda^{\kappa}(\varphi)} *(\varphi, \alpha)$

- $\mathbf{B}(\kappa, i)=\max _{\left\{I \mid \lambda^{\kappa}(I)=i\right\}} \kappa(I)$, if $i>0$; and $\mathbf{B}(\kappa, 0)=-1$. 
The main idea of this definition is to localize the change to the concerned level of beliefs. This is the aim of $\kappa^{o}$, that does the transmutation only on the projection on the concerned level. Then the result is incorporated in the full Transfinite OCF $\kappa$, with the four points of the main definition. The first point ensures that worlds in higher levels of beliefs are not moved during the change. The second point says similarly that worlds that are in lower levels of beliefs, and that are not involved in the change at the concerned level of belief, are not moved during the change. The fourth point just encode the changes on the concerned level of beliefs. The interesting part of the definition is given by the third point that says that if there are new worlds that are possible (i.e. such that $\kappa^{o}(I)=0$ ) after the transmutation on the concerned level, then they are downgraded to the lower level of beliefs. The problem is then to know where to put them in the lower level. In order to ensure minimal change for this lower level we have to try to modify as little as possible the structure of that level. This can be done by including the downgraded worlds as the least plausible worlds of this level (this is the aim of the function $\mathbf{B}$ that allows to find the plausibility of the least plausible worlds in a given level of beliefs).

Let us see this on the example.

Example 5. Suppose that we just bought a new car with new driving assistance systems, that make us remove from our driving behaviour rules that $s l \rightarrow m$. So to make this contraction we make a relative 0 -conditionalization

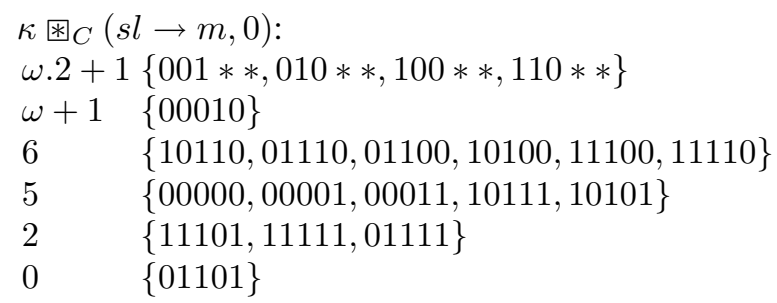

An interesting point to note is that after this relative conditionalization, the formula

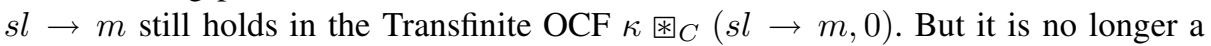
formula of the second level of belief (i.e. $\left[\kappa \otimes_{C}(s l \rightarrow m, 0)\right]_{\downarrow 2}$ ). It is now a formula of the first level of belief. So now a change in the first level of belief can remove this rule from the beliefs of the agent, whereas it was not possible before since beliefs of the second level of beliefs can not be changed by revision of the first level.

Note in particular that, to remove completely this formula from the beliefs of the agent, one has to do one more contraction:

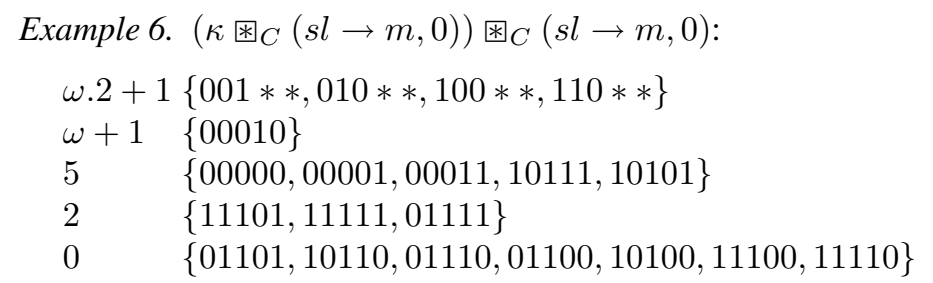

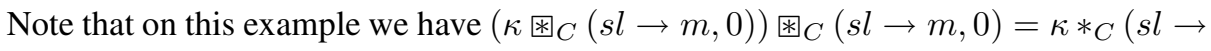
$m, 0)$. But this is generally not the case. 
As explained in the introduction, it makes sense to use different revision operators for the different levels of beliefs. For instance the less important level, that usually contains factual information can use more drastic revision operators, since loss of information in this level is not that important (with respect to loss in higher levels).

So this means that we need some adaptative change operators. We define such operators as relative transmutations where the computation of $\kappa^{o}$ depends of the level of the new piece of information:

Definition 12. Let $\kappa$ be a Transfinite OCF with n levels of beliefs. Let $\mathcal{A}=\left\{*_{1}, \ldots, *_{n}\right\}$ be a vector of $n$ absolute transmutations. Let $\alpha$ be an ordinal $\alpha<\omega$. Then the corresponding adaptative relative transmutation $\mathbf{⿴ 囗}^{\mathcal{A}}$ is defined as:

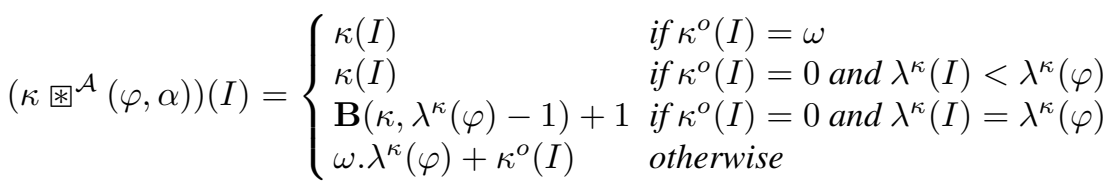

where

- $\kappa^{o}=\kappa_{\downarrow \lambda^{\kappa}(\varphi)} *_{\lambda^{\kappa}(\varphi)}(\varphi, \alpha)$

- $\mathbf{B}(\kappa, i)=\max _{\left\{I \mid \lambda^{\kappa}(I)=i\right\}} \kappa(I)$, if $i>0$; and $\mathbf{B}(\kappa, 0)=-1$.

\section{Conclusion}

In this paper we have investigated how to represent and change beliefs of an agent that are hierarchized through several levels of beliefs, where each level appears as integrity constraint for less important levels. We have shown how to represent these levels by using Ordinal Conditional Functions. This is the first time, as far as we know, that the use of transfinite ordinals is investigated. Spohn in a footnote of [14] says:

"It would be a natural idea to restrict the range of OCFs to the set of natural numbers. In fact, much of the following could thereby be simplified since usual arithmetic is simpler than the arithmetic on ordinals. For the sake of formal generality I do not impose this restriction. But larger ranges may be intuitively needed. For example, it is tempting to use OCFs with larger ranges to represent the stubbornness with which some beliefs are held in the face of seemingly arbitrarily augmentable counter-evidence."

So in this work we have proposed a representation of these stubbornly held beliefs by mean of levels of beliefs. And, more importantly, we have discussed the inadequacy of usual (absolute) transmutations to realize the change on these OCFs. So we have proposed the definition of relative transmutations, that limit the change to the concerned level of belief.

We are convinced that several other interesting change operators can be defined in the framework of Transfinite OCFs. We let this for future work. 
Acknowledgements. The author would like to thank the anonymous reviewers for their useful comments.

\section{References}

1. Alchourrón, C.E., Gärdenfors, P., Makinson, D.: On the logic of theory change: Partial meet contraction and revision functions. Journal of Symbolic Logic 50, 510-530 (1985)

2. Boutilier, C.: Iterated revision and minimal change of conditional beliefs. Journal of Philosophical Logic 25(3), 262-305 (1996)

3. Darwiche, A., Pearl, J.: On the logic of iterated belief revision. Artificial Intelligence 89, 1-29 (1997)

4. Dubois, D.: Three scenarios for the revision of epistemic states. Journal of Logic and Computation 18(5), 721-738 (2008)

5. Dubois, D., Lang, J., Prade, H.: Possibilistic logic. In: Handbook of Logic in Artificial Intelligence and Logic Programming, vol. 3, pp. 439-513. Oxford University Press, Oxford (1994)

6. Friedman, N., Halpern, J.Y.: Belief revision: a critique. In: Proceedings of the Fifth International Conference on Principles of Knowledge Representation and Reasoning (KR 1996), pp. 421-431 (1996)

7. Gärdenfors, P.: Knowledge in flux. MIT Press, Cambridge (1988)

8. Herzig, A., Rifi, O.: Update operations: a review. In: Proceedings of the Thirteenth European Conference on Artificial Intelligence (ECAI 1998), pp. 13-17 (1998)

9. Lang, J., Delgrande, J.P., Dubois, D.: Iterated revision as prioritized merging. In: Proceedings of the tenth International Conference on Principles of Knowledge Representation and Reasoning (KR 2006), pp. 210-220 (2006)

10. Jin, Y., Thielscher, M.: Iterated belief revision, revised. Artificial Intelligence 171, 1-18 (2007)

11. Katsuno, H., Mendelzon, A.O.: On the difference between updating a knowledge base and revising it. In: Proceedings of the Second International Conference on Principles of Knowledge Representation and Reasoning (KR 1991), pp. 387-394 (1991)

12. Katsuno, H., Mendelzon, A.O.: Propositional knowledge base revision and minimal change. Artificial Intelligence 52, 263-294 (1991)

13. Meyer, T.: On the semantics of combination operations. Journal of Applied Non Classical Logics 11(1/2), 59-84 (2001)

14. Spohn, W.: Ordinal conditional functions: A dynamic theory of epistemic states. In: Harper, W.L., Skyrms, B. (eds.) Causation in Decision: Belief Change and Statistics, pp. 105-134. Kluwer, Dordrecht (1988)

15. Spohn, W.: Ranking Functions, AGM Style. Internet Festschrift for Peter Gärdenfors, Lund (1999)

16. Williams, M.A.: Transmutations of knowledge systems. In: Proceedings of the Fourth International Conference on the Principles of Knowledge Representation and Reasoning (KR 1994), pp. 619-629 (1994) 